\title{
The Clinical Significance of Serum IL-33 and sST2 Alterations in the Post-Stroke Depression
}

\author{
Meirong $\mathrm{Xu}$ \\ Ganlin Wu $\mathbb{D}^{2,3}$ \\ 'Department of Geriatrics, The Second \\ Affiliated Hospital of Hubei University of \\ Science and Technology, Xianning, \\ 437100, Hubei Province, People's \\ Republic of China; ${ }^{2}$ Department of \\ Medicine, School of Clinical Medicine \\ Sciences, Hubei University of Science and \\ Technology, Xianning, 437100, Hubei \\ Province, People's Republic of China; \\ ${ }^{3}$ National Demonstration Center for \\ Experimental General Medicine \\ Education (Hubei University of Science \\ and Technology), Xianning, 437/00, \\ Hubei Province, People's Republic of \\ China
}

Introduction: This study was to test whether the serum levels of IL-33 and sST2 are correlated with the development of depression after acute ischemic stroke.

Methods: Patients diagnosed with acute ischemic stroke were selected. This study took the 24-item Hamilton Depression Rating Scale (HAMD) (score $\geq 20$ ) as the diagnostic criteria for depression. On the 21 st day after admission, patients who met the depression diagnostic criteria were included in the depression group, and patients who failed to meet the diagnostic criteria were included in the non-depression group. The serum levels of IL-33, sST2 and hsCRP were measured by enzyme-linked immunosorbent assay (ELISA).

Results: On 1st day after stroke, compared with the non-depression group, there was no significant difference in the serum IL-33, sST2 and hsCRP levels in the depression group; on 21st day after stroke, compared with the non-depression group, the serum IL-33 and hsCRP levels were significantly increased, while the SST2 level was significantly decreased in the depression group. Correlation analysis showed that IL-33 was positively correlated with the depression quantitative score and hsCRP, while sST2 was negatively correlated with the depression quantitative score and hsCRP. Regression analysis showed that IL-33 and sST2 were independent risk factors for the depression after acute ischemic stroke.

Discussion: The abnormal alterations of serum IL-33 and sST2 levels in the stroke patients may serve as one of the risk factors for the occurrence and exacerbation of the depression, and its mechanism may be related to the promotion of inflammatory factor production in vivo.

Keywords: IL-33, sST2, post-stroke depression, PSD, clinical significance, risk factor

\section{Introduction}

Depression is a kind of mood disorder, characterized by unresponsiveness, apathy, self-blame, self-guilt, and sleep disorders. The prevalence of post-stroke depression (PSD) is high, reported to be as high as $39-52 \%$ within 5 years. ${ }^{1}$ Depression will significantly increase the recurrence and mortality rates of stroke patients. ${ }^{2,3}$ Furthermore, many factors make the diagnosis of PSD difficult, such as the different occurrence time of $\mathrm{PSD}^{4}{ }^{4}$ the inconspicuous symptoms of depression, and the language impairment in some patients. Currently, there is no uniform diagnostic standard, ${ }^{5}$ and it can very easily be misdiagnosed. ${ }^{6}$ The pathogenesis of PSD may be associated with various factors, and it is not fully understood. Therefore, exploring the mechanism is of great urgent for the early diagnosis and treatment of PSD. Previous studies on the underlying mechanisms have provided support for the neuroinflammatory hypothesis in PSD. For example, several studies have shown that IL-6 and IL-18 may be participated in the etiology and progression
Department of Medicine, School of Clinical Medicine Sciences, Hubei University of Science and Technology, No. 88 Xianning Road, Xianning, 437100, Hubei Province, People's Republic of China

Tel +86-07I5-8102568

Email wu_ganlin65@2Icn.com 
of PSD. ${ }^{7,8}$ Since IL-18 and IL-6 have been considered to be associated with inflammatory responses at lesion site and detrimental outcomes of stroke, these two inflammatory factors have attracted the attention of scholars in connection with PSD. IL-33 is a recently discovered member of interleukin family. Soluble ST2 (sST2) is the receptor of IL-33, which binds to IL-33 and blocks its activity. ${ }^{9}$ Serum levels of IL-33 and sST2 have been shown to be related to neuroinflammation. In contrast to the immunomodulation effect of IL-18 which can stimulate Th1 cells to secrete a variety of cytokines and promote the proliferation of Th1 cells, IL-33/sST2 can regulate Th1/Th2 balance by promoting Th2 anti-inflammatory immunity and inhibiting Th1 immune response. ${ }^{9}$ Emerging studies have revealed that IL-33/sST2 is closely correlated with cardiovascular diseases, autoimmune and nervous system diseases, ${ }^{10-12}$ yet its role in the nervous system remains poorly understood. Some studies have demonstrated that serum IL-33 has a protective effect against stroke through anti-inflammatory and can improve the prognosis of stroke. $^{13,14}$ However, its effect on the pathogenesis of PSD remains to be explored. Therefore, in this study, we investigated the serum IL-33 and sST2 expression in the patients with PSD, and evaluated their clinical significance.

\section{Materials and Methods}

\section{Patients}

From December 2017 to December 2019, patients with acute ischemic stroke diagnosed by CT or MRI at the Second Affiliated Hospital of Hebei University of Science and Technology were selected. All patients were admitted to the hospital within 24 hours after the onset of illness. The extent of neurologic deficits was measured by the NIH Stroke Scale/Score (NIHSS) and graded as follows: $1-4$ as mild, $5-15$ as moderate, and $>16$ as severe. On the 21 st day after admission, two psychiatrists assessed the depressive symptoms of the patients. According to the criteria of the American Diagnostic and Statistical Manual for Mental Disorders (the fourth edition), a 24-item HAMD score was used to determine whether a patient had depression and its severity. A score $>20$ was considered as the diagnostic criteria for depression, and patients who met the criteria were included into the depression group, while other patients were included into the nondepression group. The severity of depression was classified as: severe: $\geq 35$; mild to moderate: $21-34$. The excluded patients included those who were taking antidepressants or medications affecting depression scale scores 2 weeks prior to admission, those who were taking NSAIDs, corticosteroids, antibiotics, immunomodulators, etc. 2 weeks prior to admission, those who could not cooperate with clinical psychological testing due to aphasia or dementia, etc., and those with psychiatric disorders, central nervous system diseases, heart failure, history of trauma, history of surgery, hepatic and renal insufficiency, severe infections and Th2-related diseases (eg, asthma, non-specific dermatitis, allergic reactions, etc.). Finally, a total of 160 patients were enrolled in this study, including 100 non-depressed patients (non-depressed group) and 60 patients with PSD (depressed group). In the depression group, there were 46 cases of mild to moderate and 14 cases of severe depression. The study was approved by The Second Affiliated Hospital of Hubei University of Science and Technology ethical review committee and all participants signed an informed consent form. This study was conducted in accordance with the Declaration of Helsinki.

\section{Blood Sample Collection and Processing}

All subjects had at least 12 hours of fasting before blood collection on the 1st and 21st days after the admission. Blood samples were processed in a centrifuge at $3000 \mathrm{rpm}$ for $10 \mathrm{~min}$. Plasma was collected and stored at $-80^{\circ} \mathrm{C}$ until analysis. On the 1st day after admission, the fasting blood glucose (FBG), creatinine, and glutamic-pyruvic transaminase were detected by spectrophotometry using the Abbott Aeroset 2.0 (Abbott Diagnostic, Illinois, USA). On the 1st and 21 st days after admission, IL-33, sST2 and hsCRP levels were assayed by a commercially available competitive enzyme-linked immunoassay (ELISA) kit (MyBioSource, Catalog Number: MBS756338, Inc. San Diego, CA, USA). To minimize the assay variance, all samples were analyzed in duplicate on the same day in a random order by a technician blinded to the clinical diagnosis. The coefficients of intra- and inter-assay variation were $<6 \%$. All steps were performed strictly in accordance with the instructions of the kit.

\section{Statistical Analysis}

SPSS 19.0 was used to perform statistical analysis. Data of the normal distribution were expressed as mean \pm deviation $(\mathrm{X} \pm \mathrm{s})$, and the $t$-test was used to make comparisons between the two groups. Paired samples $t$-test was applied to compare the serum concentrations of inflammatory 
cytokines on day 1 and day 21. Numerical data were described as $\mathrm{n} / \%$ and the Chi-square tests were used. Correlation analysis was performed using the Spearman and Pearson methods. Risk factors for PSD were determined by univariate and multivariate logistic regression analysis. Forward conditional logistic regression which included all factors with significant differences in univariate analysis was used to find independent risk factors for PSD. A $p$-value $<0.05$ was considered statistically significant with a confidence interval of $95 \%$.

\section{Results}

\section{Comparison of the Baseline Characteristics Between Non-Depression Group and Depression Group}

A total of 160 patients were included in this study. In addition to inflammatory factors, factors that may affect PSD included gender, age, lesion site, degree of neurological deficit, blood pressure, and blood sugar. In our present study, there were no significant differences between the non-depression group and depression group in terms of gender, age, and lesion location (all $P>0.05$, Table 1). Significant differences were found in

Table I Intergroup Comparison of Baseline Characteristics

\begin{tabular}{|c|c|c|c|c|}
\hline Factors & $\begin{array}{l}\text { Non-Depression } \\
\text { Group } \\
(n=100)\end{array}$ & $\begin{array}{l}\text { Depression } \\
\text { Group } \\
(n=60)\end{array}$ & $t / x 2$ & $p$ \\
\hline $\begin{array}{l}\text { Sex }(n / \%) \\
\text { Male } \\
\text { Female }\end{array}$ & $\begin{array}{l}37 / 46.3 \\
43 / 53.7\end{array}$ & $\begin{array}{l}32 / 53.3 \\
28 / 46.7\end{array}$ & 0.688 & 0.407 \\
\hline Age ( $x \pm s$, year) & $52.12 \pm 10.73$ & $48.6 \pm 11.46$ & 0.98 & 0.382 \\
\hline $\begin{array}{l}\text { Lesion site (n/\%) } \\
\text { Left side } \\
\text { Both side }\end{array}$ & $\begin{array}{l}30 / 37.5 \\
9 / 11.3\end{array}$ & $\begin{array}{l}28 / 46.7 \\
5 / 8.3\end{array}$ & 1.26 & 0.532 \\
\hline $\begin{array}{l}\text { Degree of } \\
\text { neurological } \\
\text { deficit (n/\%) } \\
\text { Mild } \\
\text { Moderate } \\
\text { Severe }\end{array}$ & $\begin{array}{l}50 / 62.5 \\
25 / 31.3 \\
5 / 6.3\end{array}$ & $\begin{array}{l}12 / 20 \\
32 / 53.3 \\
16 / 26.7\end{array}$ & 27.62 & 0.000 \\
\hline $\begin{array}{l}\text { Blood pressure } \\
\text { (n/\%) } \\
\text { Normal } \\
\text { High }\end{array}$ & $\begin{array}{l}46 / 57.7 \\
34 / 42.5\end{array}$ & $\begin{array}{l}20 / 33.3 \\
40 / 66.7\end{array}$ & 8.036 & 0.005 \\
\hline $\begin{array}{l}\text { Blood sugar(n/\%) } \\
\text { Normal } \\
\text { Diabetes }\end{array}$ & $\begin{array}{l}49 / 61.3 \\
31 / 38.7\end{array}$ & $\begin{array}{l}26 / 43.3 \\
34 / 56.7\end{array}$ & 4.425 & 0.035 \\
\hline
\end{tabular}

the degree of neurological deficits, blood pressure, and blood sugar between the two groups (all $P<0.05$ ).

\section{Inter- and Intra-Group Comparisons of Inflammatory Factors at the Ist and 2 Ist Day}

On the 1st day after stroke, there was no substantial difference in serum IL-33, ST2 and hsCRP levels between the non-depression and depression groups $(P>0.05$, Table 2, Figure $1 \mathrm{~A}-\mathrm{C}$ ). Whereas on the $21 \mathrm{st}$ day, serum IL-33 and hsCRP levels were significantly higher, while serum sST2 levels were significantly lower in the depression group than those in the non-depression group $(P<0.01$, Figure $1 \mathrm{~A}-\mathrm{C})$. In the non-depression group, there were no substantial differences in serum IL-33, ST2 and hsCRP levels post 21 days. In contrast, in the depression group, IL-33 and hsCRP levels were significantly increased and sST2 levels were significantly decreased after 21 days $(P<0.01$, Figure $1 \mathrm{~A}-\mathrm{C})$.

\section{Comparison of Inflammatory Factors in the Groups with Different Degrees of Depression on the 2 Ist Day}

On the 21st day after stroke, the levels of IL-33 were positively correlated with the severity of depression $(P<0.05$ or $P<0.01$, Table 3, Figure 2), while the levels of $\mathrm{sST} 2$ were inversely correlated with the severity of depression $(P<0.05$ or $P<0.01$, Table 3 , Figure 2$)$. There was no substantial difference in the levels of serum hsCRP between the two groups ( $P>0.05$, Table 3 , Figure 2$)$.

Table 2 Inter- and Intra-Group Comparisons of Inflammatory Factors at the Ist and 2 Ist Day

\begin{tabular}{|c|c|c|c|c|}
\hline Factors & $\begin{array}{l}\text { Non- } \\
\text { Depression } \\
\text { Group } \\
(n=100)\end{array}$ & $\begin{array}{l}\text { Depression } \\
\text { Group } \\
(n=60)\end{array}$ & $\mathbf{t}$ & $p$ \\
\hline \multicolumn{5}{|l|}{ IL-33 } \\
\hline Ist day & $16.04 \pm 7.18$ & $|7.23 \pm 5.6|$ & & \\
\hline 2 Ist day & $14.69 \pm 6.24$ & $37.89 \pm 14.58$ & -11.55 & 0.001 \\
\hline \multicolumn{5}{|l|}{ sST2(ng/mL) } \\
\hline Ist day & $8.82 \pm 3.15$ & $9.01 \pm 3.26$ & & \\
\hline 2 Ist day & $8.29 \pm 2.91$ & $4.23 \pm 2.04$ & 9.71 & 0.01 \\
\hline \multicolumn{5}{|l|}{ hsCRP(mg/L) } \\
\hline Ist day & $2.02 \pm 0.96$ & $2.38 \pm 1.03$ & & \\
\hline 21 st day & $1.92 \pm 1.03$ & $4.60 \pm 1.18$ & -8.94 & 0.01 \\
\hline
\end{tabular}



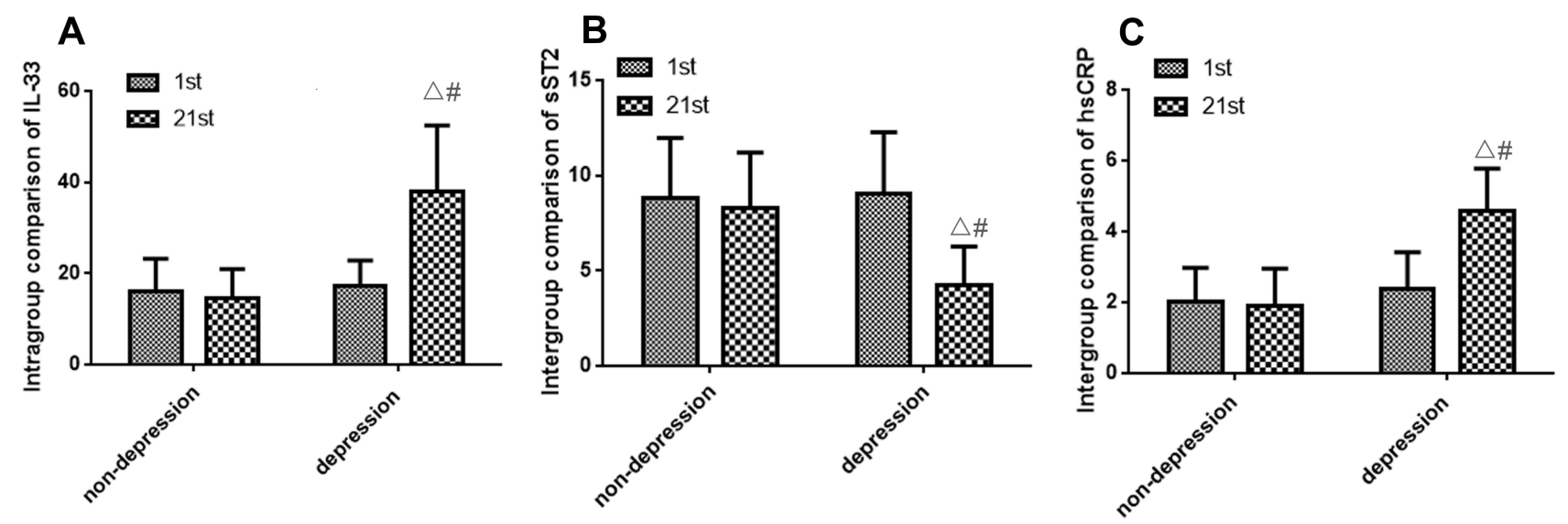

Figure I Inter- and intra-group comparisons of inflammatory factors at the Ist and 2 Ist day. (A-C) Intra-group comparisons of inflammatory factors IL-33, sST2, and hsCRP at the Ist and 2 Ist day.

Notes: ${ }^{\#} p<0.01$ for comparison between non-depression and depression group; ${ }^{\Delta} p<0.0$ I for comparison between Ist and 2 Ist day

\section{Correlation Analysis of IL-33 and sST2 Levels with hsCRP and HAMD Scores}

Spearman correlation analysis showed that serum hsCRP levels were positively correlated with HAMD score, and serum IL-33 levels were positively correlated with HAMD scores and hsCRP; serum sST2 levels were negatively correlated with HAMD scores and hsCRP $(P<0.001)$. See Table 3.

\section{Multivariate Logistic Regression Analysis of Depression in Stroke Patients}

Taking depression in patients with acute stroke as the dependent variable, the 24-items HAMD score $<20$ was assigned as 0 and $>20$ as 1 ; logistic regression analysis was performed for the related factors with significant differences in univariate analysis results, and the independent variables were IL-33, sST2, hsCRP, NIHSS score, blood pressure and blood sugar. No diabetes was assigned as 0 , diabetes was assigned as 1 ; normal blood pressure was assigned as 0 , hypertension was assigned as 1 ; binary logistic regression analysis was used in this study.

Table 3 Comparison of Inflammatory Factors in Groups with Different Degrees of Depression

\begin{tabular}{|l|l|l|}
\hline Factors & $\begin{array}{l}\text { Mild to Moderate Group } \\
(\mathbf{n = 2 4 )}\end{array}$ & $\begin{array}{l}\text { Severe Group } \\
(\mathbf{n}=1 \mathbf{1 4})\end{array}$ \\
\hline IL-33 & $38.73 \pm 18.03$ & $44.93 \pm 11.36^{\#}$ \\
sST2 & $4.27 \pm 1.31$ & $2.57 \pm 0.93^{\#}$ \\
hsCRP & $3.59 \pm 0.88$ & $4.19 \pm 1.04$ \\
\hline
\end{tabular}

Notes: ${ }^{\#} p<0.01$ for comparison between Mild to moderate group and Severe group.
Regression analysis indicated that blood pressure, NIHSS score, IL-33 and hsCRP were independent risk factors for PSD, and $\mathrm{SST} 2$ was an independent protective factor $(P<0.05)$, as shown in Tables 4 and 5 .

\section{Discussion}

Our results revealed that the more severe the neurological impairment, the higher was the incidence of PSD. We also observed the association between hypertension and PSD occurrence, which is consistent with the results of previous studies. ${ }^{15,16}$ Meanwhile, we did not find an association between the different lesion sites (left, right or bilateral) and the occurrence of depression. Moreover, in our study, we found that the levels of serum hsCRP in stroke patients with PSD were significantly higher than those without depression, and the serum hsCRP levels were positively correlated with HAMD scores. These results supported the increased inflammation reaction in the patients with PSD. The impact of certain inflammatory markers on PSD, such as TNF and hsCRP, has also been explored by various scholars. ${ }^{16-18}$ Cheng et $\mathrm{al}^{16}$ have proposed that the elevated serum hsCRP and homocysteine levels are closely related to the risk of PSD within one year after the onset of acute ischemic stroke, and suggested that combining these two indicators could provide an early prognosis assessment of PSD.

Interleukins are known to play important roles in activating and regulating immune cells and participating in the inflammatory response. IL-33 is a member of the IL-1 family. Compared to other members of IL-1 family, the physiological function of IL-33 may be more complex. It can promote the anti-inflammatory immunity of Th2 and 

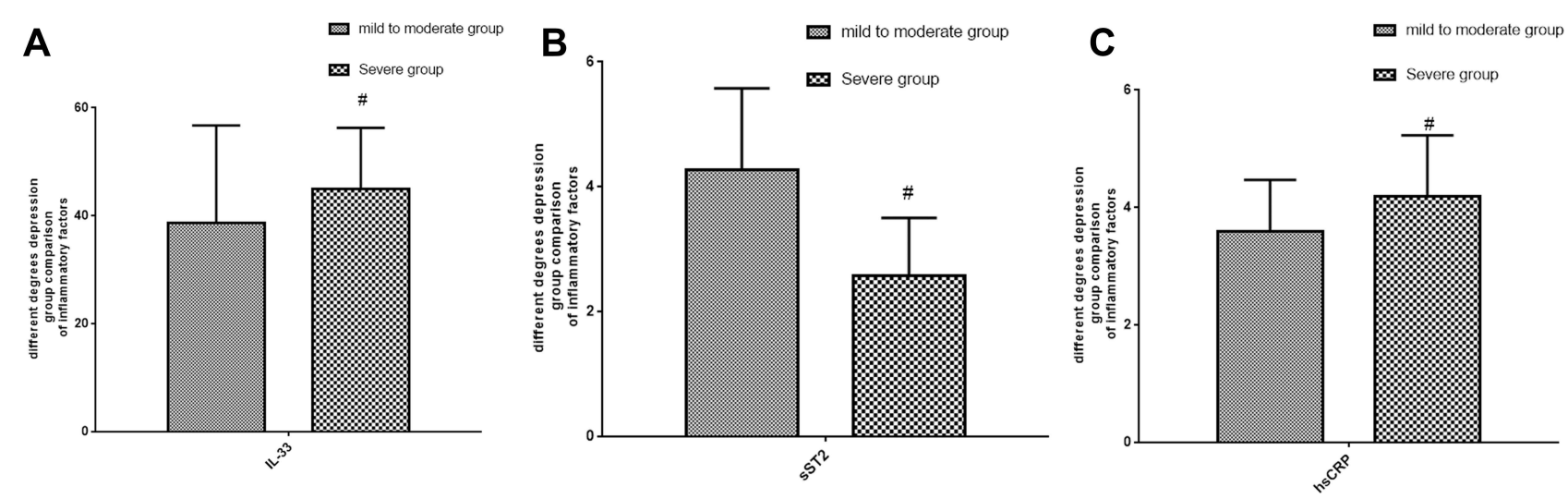

Figure 2 Comparison of inflammatory factors in the groups with different degrees of depression on the 2 Ist day. (A-C) Comparison of inflammatory factors IL-33, sST2, and hsCRP in the groups with different degrees of depression on the 21 st day.

Notes: ${ }^{\#} p<0.01$ for comparison between mild to moderate and Severe groups.

inhibit the immune response of Th1 $;^{9}$ meanwhile, IL-33 can act as a signaling molecule to bind to the transmembrane ST2 (ST2L). Under the stimulation of IL-33, the p38 MAPK pathway was found to activate bone marrow-derived mast cells to produce inflammatory factors such as IL-13, IL-6, $\mathrm{TNF}$, and GM-CSF $;^{19,20} \mathrm{Ali}^{21}$ has found that the overexpression of IL-33 led to the reduced and delayed expression of endogenous NF- $\mathrm{kB}$ target genes stimulated by rhil- $1 \beta$, and reduced the genes triggered by NF- $\kappa \mathrm{B}$. Thus, IL-33 has the dual pro-inflammatory or anti-inflammatory properties. In stroke patients, several studies have indicated that serum IL33 has anti-inflammatory protective effects, which may improve the prognosis of stroke. ${ }^{13,22}$ In the experimental study of Zhang et al, ${ }^{23}$ recombinant IL-33 indeed presented protective effects, enhancing the Th2 type, antiinflammatory immunological response in the brain, inhibited microglial activation, and depleted cytotoxic $\mathrm{T}$ cells, along with the increase of IL-10 production. In contrast, in the study of Li et al, ${ }^{24}$ IL-33 proved to be significantly elevated after acute ischemic stroke, and high IL-33 levels were associated with worse functional outcomes. Surprisingly, IL-33 showed an inverse correlation with the infarction volume and was higher in mild than in severe lesions (NIHSS score). For instance, Kempuraj et al ${ }^{25}$ mixed neurons with glial maturation factor (GMF) with or without IL-

Table 4 Correlation Analysis of IL-33 and sST2 Levels with hsCRP and HAMD Score

\begin{tabular}{|l|l|l|l|l|l|l|}
\hline \multirow{2}{*}{ Index } & \multicolumn{2}{|c|}{ IL-33 } & \multicolumn{2}{c|}{ sST2 } & \multicolumn{2}{c|}{ hsCRP } \\
\cline { 2 - 7 } & $\boldsymbol{r}$ & $\boldsymbol{p}$ & $\boldsymbol{r}$ & $\boldsymbol{p}$ & $\boldsymbol{r}$ & $\boldsymbol{p}$ \\
\hline HAMD score & 0.785 & 0.001 & -0.660 & 0.001 & 0.620 & 0.001 \\
hsCRP & 0.587 & 0.001 & -0.467 & 0.001 & 1 & \\
\hline
\end{tabular}

33 in vitro and subsequently found that GMF induced IL-33 release, which enhanced the release of astrocytic tumor necrosis factor- $\alpha$ (TNF- $\alpha$ ) in mice. And astrocytes are thought to be closely associated with the development of PSD. ${ }^{26,27}$ There are as well some studies on the effects of IL33 on cognitive function, ${ }^{28,29}$ which found that IL-33 may lead to cognitive dysfunction in experimental cerebral malaria and Alzheimer's disease. From the previous data, we know that it is controversial how IL-33 affects the central nervous system disorders. Therefore, the role of IL-33 and sST2 levels in depression of stroke patients have attracted our attention. Moreover, its mechanism is not well understood. For that reason, in our study, we investigated the alterations of IL-33/sST2 levels of patients with PSD.

In this study, we found that IL-33 levels were increased and SST2 levels were decreased in PSD patients on the 21st day after stroke. As depression exacerbated, the changes tended to become more obvious, suggesting that the abnormal increase in IL-33 may promote the onset and development of PSD, while sST2 can competitively bind IL33, blocking the binding of IL-33/ST2L and alleviating the inflammatory reaction. However, previous findings of the protective effect of IL-33 in stroke patients seem to

Table 5 Multivariate Logistic Regression Analysis of Depression in Stroke Patients

\begin{tabular}{|l|l|l|l|l|l|l|}
\hline Factors & $\boldsymbol{\beta}$ & SE & OR & $\mathbf{9 5 \%} \mathbf{C l}$ & wals & $\boldsymbol{p}$ \\
\hline Blood pressure & 2.97 & 1.28 & 19.50 & $1.58 \sim 240.88$ & 5.36 & 0.02 \\
Blood sugar & -1.29 & 1.09 & 0.28 & $0.03 \sim 2.34$ & 1.39 & 0.24 \\
NIHSS score & 0.24 & 0.12 & 1.27 & $1.01 \sim 1.61$ & 4.10 & 0.04 \\
IL33 & 0.31 & 0.09 & 1.36 & $1.14 \sim 1.63$ & 11.58 & 0.001 \\
SST2 & -1.05 & 0.34 & 0.35 & $0.18 \sim 0.69$ & 4.8 & 0.002 \\
hsCRP & 1.07 & 0.49 & 2.91 & $1.12 \sim 7.58$ & 5.676 & 0.03 \\
\hline
\end{tabular}


paradoxically contradict our results. We believe that the reason may be related to the complex biological effects of IL-33/ST2 signaling pathway. Since this pathway has a twoway effect on many diseases, it is a double-edged sword. ${ }^{30}$ Besnard et $\mathrm{al}^{31}$ suggested that IL-33 exerted beneficial or detrimental effects directly or indirectly through a series of downstream substances, and its multipotency depended on the disease context or cell environment. Therefore, according to our results, IL-33 in the low concentration range is likely to exhibit anti-inflammatory effects in stroke patients, whereas IL-33 in the high concentration range may play a pro-inflammatory role in PSD. The biological microenvironment and the downward signaling pathways may play a major role in the final shape of IL-33 effects. The IRAK1, IRAK4, and TRAF6 mediated selective activation of NFkB, JNK, ERK may fine-tune the outputs. ${ }^{32}$ There is a crosstalk between NFkB, IFNg, and TNFa signaling, and it is known that these two cytokines interfere with glutamatergic signaling, as it synthesized in a recent review. ${ }^{33}$

Correlation analysis showed that serum IL-33 was positively correlated with HAMD score and hsCRP, while serum sST2 was negatively correlated with HAMD score and hsCRP, suggesting that the mechanism of IL-33/ST2 signaling pathway in PSD patients may be related to the production of inflammatory factors in vivo. Furthermore, regression analysis showed that IL-33 was an independent risk factor for PSD, and $\mathrm{SST} 2$ was an independent protective factor for PSD, indicating that serum IL-33 and sST2 can be used as clinical predictors of PSD.

\section{Conclusion}

In conclusion, the results of this study suggest that the abnormal alterations of IL-33 and sST2 in acute stroke patients may contribute to the onset and aggravation of depression, and the mechanism may be associated with facilitating the production of inflammatory factors. Our study provides us a new molecular mechanism of PSD from the perspective of IL-33/sST2 signaling pathway. However, our study has certain limitations. The main limits were the small size of samples and the fact that only patients with depression within three weeks after stroke were included in the study. These limitations have led to the restrictions in their application. The findings of our work need to be validated with more patients and a longer follow-up duration. More basic and clinical studies are required to investigate the specific molecular mechanisms of IL-33/ST2 signaling pathway in the pathogenesis of PDS.

\section{Abbreviations}

HAMD, Hamilton Depression Rating Scale; ELISA, enzyme-linked immunosorbent assay; PSD, post-stroke depression; sST2, Soluble ST2; NIHSS, NIH Stroke Scale/Score; ST2L, transmembrane ST2; GMF, glial maturation factor; TNF- $\alpha$, tumor necrosis factor- $\alpha$.

\section{Ethics Approval and Consent to Participate}

The study was approved by The Second Affiliated Hospital of Hubei University of Science and Technology ethical review committee and all participants signed an informed consent form. This study was conducted in accordance with the Declaration of Helsinki.

\section{Author Contributions}

All authors made a significant contribution to the work reported, whether that is in the conception, study design, execution, acquisition of data, analysis and interpretation, or in all these areas; took part in drafting, revising or critically reviewing the article; gave final approval of the version to be published; have agreed on the journal to which the article has been submitted; and agree to be accountable for all aspects of the work.

\section{Funding}

There is no funding to report.

\section{Disclosure}

There are no potential conflicts of interest to disclose.

\section{References}

1. Robinson RG, Jorge RE. Post-stroke depression: a review. Am $J \quad$ Psychiatry. 2016;173:221-231. doi:10.1176/appi.ajp.201 5.15030363

2. Villa RF, Ferrari F, Moretti A. Post-stroke depression: mechanisms and pharmacological treatment. Pharmacol Ther. 2018;184:131-144. doi:10.1016/j.pharmthera.2017.11.005

3. Cai W, Mueller C, Li YJ, Shen WD, Stewart R. Post stroke depression and risk of stroke recurrence and mortality: a systematic review and meta-analysis. Ageing Res Rev. 2019;50:102-109. doi:10.1016/j. arr.2019.01.013

4. Guiraud V, Gallarda T, Calvet D, et al. Depression predictors within six months of ischemic stroke: the DEPRESS study. Int $J$ Stroke. 2016;11:519-525. doi:10.1177/1747493016632257

5. Kouwenhoven SE, Kirkevold M, Engedal K, Kim HS. Depression in acute stroke: prevalence, dominant symptoms and associated factors. Disabil Rehabil. 2011;33:539-556. doi:10.3109/09638288.20 10.505997

6. Naghavi FS, Koffman EE, Lin B, Du J. Post-stroke neuronal circuits and mental illnesses. Int $J$ Physiol Pathophysiol Pharmacol. 2019;11:1-11. 
7. Zhang XF, Zou W, Yang Y. Effects of IL-6 and cortisol fluctuations in post-stroke depression. J Huazhong Univ Sci Technolog Med Sci. 2016;36(5):732-735. doi:10.1007/s11596-016-1653-0

8. Kang HJ, Bae KY, Kim SW, et al. Effects of interleukin-6, interleukin-18, and statin use, evaluated at acute stroke, on post-stroke depression during 1-year follow-up. Psychoneuroendocrinology. 2016;72:156-160. doi:10.1016/j. psyneuen.2016.07.001

9. Vocca L, Di Sano C, Uasuf CG, et al. IL-33/ST2 axis controls Th2/ IL-31 and Th17 immune response in allergic airway diseases. Immunobiology. 2015;220:954-963. doi:10.1016/j.imbio.2015.02.005

10. Yang J, Huang X, Hu F, Fu X, Jiang Z, Chen K. LncRNA ANRIL knockdown relieves myocardial cell apoptosis in acute myocardial infarction by regulating IL-33/ST2. Cell Cycle. 2019;18:3393-3403. doi:10.1080/15384101.2019.1678965

11. Du LX, Wang YQ, Hua GQ, Mi WL. IL-33/ST2 pathway as a rational therapeutic target for $\mathrm{CNS}$ diseases. Neuroscience. 2018;369:222-230. doi:10.1016/j.neuroscience.2017.11.028

12. Xu WD, Zhang M, Zhang YJ, Ye DQ. IL-33 in rheumatoid arthritis: potential role in pathogenesis and therapy. Hum Immunol. 2013;74:1057-1060. doi:10.1016/j.humimm.2013.06.029

13. Li XM, Wang XY, Feng XW, et al. Serum interleukin-33 as a novel marker for long-term prognosis and recurrence in acute ischemic stroke patients. Brain Behav. 2019;9:e01369. doi:10.1002/brb3.1369

14. Xiao W, Guo S, Chen L, Luo Y. The role of Interleukin-33 in the modulation of splenic T-cell immune responses after experimental ischemic stroke. J Neuroimmunol. 2019;333:576970. doi:10.1016/j. jneuroim.2019.576970

15. Li P, Zhang QL, Li SY. [The relationship between acute inflammatory cytokines, nerve function defect, daily living ability and PSD]. Zhongguo Ying Yong Sheng Li Xue Za Zhi. 2017;33:121-123. doi:10.12047/j.cjap.5512.2017.031. Chinese

16. Cheng LS, Tu WJ, Shen Y, Zhang LJ, Ji K. Combination of high-sensitivity C-reactive protein and homocysteine predicts the post-stroke depression in patients with ischemic stroke. Mol Neurobiol. 2018;55:2952-2958. doi:10.1007/s12035-017-0549-8

17. Mu Y, Wang Z, Zhou J, Tan C, Wang H. Correlations of post-stroke depression with inflammatory response factors. Iran J Public Health. 2018;7:988-993.

18. Yang RR, Lu BC, Li T, Du YF, Wang X, Jia YX. The relationship between high-sensitivity $\mathrm{C}$-reactive protein at admission and post stroke depression: a 6-month follow-up study. Int $J$ Geriatr Psychiatry. 2016;31:231-239. doi:10.1002/gps.4315

19. McCarthy PC, Phair IR, Greger C, et al. IL-33 regulates cytokine production and neutrophil recruitment via the p38 MAPK-activated kinases MK2/3. Immunol Cell Biol. 2019;97(1):54-71. doi:10.1111/ imcb. 12200

20. Bawazeer MA, Theoharides TC. IL-33 stimulates human mast cell release of CCL5 and CCL2 via MAPK and NF- $\kappa B$, inhibited by methoxyluteolin. Eur J Pharmacol. 2019;865:172760. doi:10.1016/ j.ejphar.2019.172760
21. Ali S, Mohs A, Thomas M, et al. The dual function cytokine IL-33 interacts with the transcription factor NF- $\mathrm{B}$ to dampen NFผB-stimulated gene transcription. J Immunol. 2011;187:1609-1616. doi:10.4049/jimmunol.1003080

22. Luo Y, Zhou Y, Xiao W, et al. Interleukin-33 ameliorates ischemic brain injury in experimental stroke through promoting $\mathrm{Th} 2$ response and suppressing Th17 response. Brain Res. 2015;1597:86-94. doi:10.1016/j.brainres.2014.12.005

23. Zhang SR, Piepke M, Chu HX, et al. IL-33 modulates inflammatory brain injury but exacerbates systemic immunosuppression following ischemic stroke. JCI Insight. 2018;3(18):e121560. doi:10.1172/jci. insight. 121560

24. Li Q, Lin Y, Huang W, et al. Serum IL-33 is a novel diagnostic and prognostic biomarker in acute ischemic stroke. Aging Dis. 2016;7:614-622. doi:10.14336/AD.2016.0207

25. Kempuraj D, Khan MM, Thangavel R, Xiong Z, Yang E, Zaheer A. Glia maturation factor induces interleukin-33 release from astrocytes: implications for neurodegenerative diseases. $J$ Neuroimmune Pharmacol. 2013;8:643-650. doi:10.1007/s11481-013-9439-7

26. Feigin VL, Forouzanfar MH, Krishnamurthi R, et al. Global and regional burden of stroke during 1990-2010: findings from the Global Burden of Disease Study 2010. Lancet. 2014;383:245-254. doi:10.1016/S0140-6736(13)61953-4

27. Keck F, Kortchak S, Bakovic A, Roberts B, Agrawal N, Narayanan A. Direct and indirect pro-inflammatory cytokine response resulting from TC-83 infection of glial cells. Virulence. 2018;9:1403-1421. doi:10.1080/21505594.2018.1509668

28. Reverchon F, Mortaud S, Sivoyon M, et al. IL-33 receptor ST2 regulates the cognitive impairments associated with experimental cerebral malaria. PLoS Pathog. 2017;13(4):e1006322. doi:10.1371/ journal.ppat.1006322

29. Liang CS, Su KP, Tsai CL, et al. The role of interleukin-33 in patients with mild cognitive impairment and Alzheimer's disease. Alzheimers Res Ther. 2020;12:86. doi:10.1186/s13195-020-00652-Z

30. Liu X, Xiao Y, Pan Y, Li H, Zheng SG, Su W. The role of the IL-33/ ST2 axis in autoimmune disorders: friend or foe? Cytokine Growth Factor Rev. 2019;50:60-74. doi:10.1016/j.cytogfr.2019.04.004

31. Besnard AG, Guabiraba R, Niedbala W, et al. IL-33-mediated protection against experimental cerebral malaria is linked to induction of type 2 innate lymphoid cells, M2 macrophages and regulatory T cells. PLoS Pathog. 2015;11:e1004607. doi:10.1371/journal.ppat.1004607

32. Liew FY, Girard J-P, Turnquist HR, et al. Interleukin 33 in health and disease. Nat Rev Immunol. 2016;16:676-689. doi:10.1038/ nri.2016.95

33. Nagy E, Frigy A, Szász JA, Horváth E. Neuroinflammation and microglia/macrophage phenotype modulate the molecular background of post-stroke depression: a literature review. Exp Therap Med. 2020;20:2510-2523.

\section{Publish your work in this journal}

The Journal of Multidisciplinary Healthcare is an international, peerreviewed open-access journal that aims to represent and publish research in healthcare areas delivered by practitioners of different disciplines. This includes studies and reviews conducted by multidisciplinary teams as well as research which evaluates the results or conduct of such teams or healthcare processes in general. The journal covers a very wide range of areas and welcomes submissions from practitioners at all levels, from all over the world. The manuscript management system is completely online and includes a very quick and fair peer-review system. Visit http://www.dovepress.com/testimonials. php to read real quotes from published authors. 